\title{
EWOD Driving of Droplet on NxM Grid Using Single-Layer Electrode Patterns
}

\author{
Shih-Kang Fan*, Peter-Patrick de Guzman, and Chang-Jin "CJ" Kim \\ Mechanical and Aerospace Engineering Department University of California, Los Angeles (UCLA) \\ Los Angeles, CA 90095-1597
}

\section{ABSTRACT}

This paper demonstrates 2-D droplet movement implementing electrowetting-on-dielectric (EWOD) for actuation. Experimentation on different electrode configurations reveals certain requirements and flexibilities for EWOD device design. The current device has a single layer of rectangular electrode rowsone set of rows on top and the other set on bottom plate, orthogonally oriented. The innovation lies in the use of only $\mathrm{N}+\mathrm{M}$ electrodes to control NxM different positions of a droplet on a 2-D grid without requiring fabrication of multi-layer electrodes.

\section{INTRODUCTION}

Recently, our group has reported how to create (from reservoir), drive, divide, or merge droplets, all using electrowetting-on-dielectric (EWOD) actuation [1]. EWOD is an actuation mechanism that is based on electrically controlling a surface's wettability to aqueous liquids [2,3]. With these four functions on individual droplets in hand, the next major challenge to realizing 2-D reconfigurable digital microfluidic circuits (Fig. 1) $[4,5]$ is how to drive individual droplets on a NxM array of possible droplet positions.

An obvious approach to drive droplets with EWOD on a $\mathrm{NxM}$ array is to have one drive electrode for every designated position in the array. Taking this approach, although certainly feasible and common in integrated circuits (IC), calls for physical connection to each individual electrode within the array independently. However, the desire to minimize fabrication complexity and the uncertainties about EWOD mechanism on nonplanar surfaces motivated our search for a simpler solution.

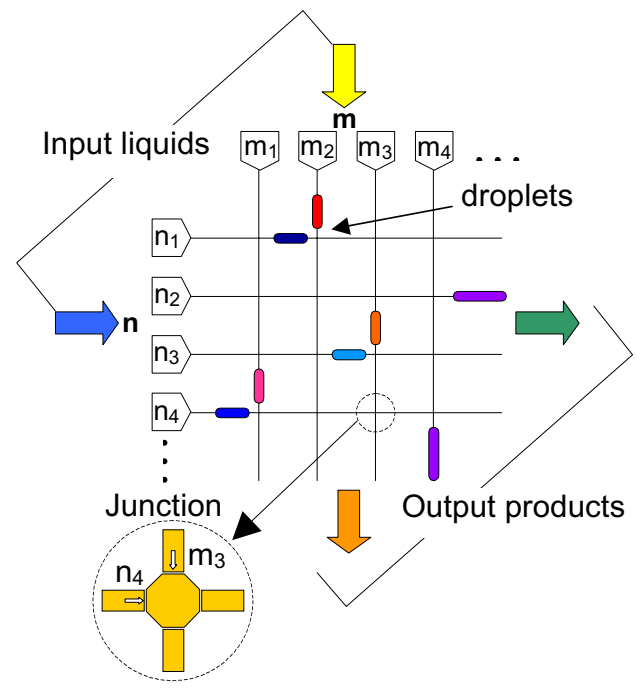

Figure 1. Conceptual description of reconfigurable digital microfluidic circuits [5].

\footnotetext{
* Travel support has been generously provided by the Transducers Research Foundation and by the DARPA MEMS and DARPA BioFlips programs.
}

\section{TECHNICAL BACKGROUND}

Fig. 2 shows the cross section and top view of an EWOD device with a single-layer of electrodes on both top and bottom glass plates [1]. A liquid droplet is placed in the gap between these two plates. Driving electrodes are patterned on one glass plate (shown bottom) subsequently coated with a dielectric layer and a thin hydrophobic coating (e.g., Teflon). The other plate has an unpatterned reference electrode layer covered with a thin hydrophobic coating. For clarification, we define the electrode providing the reference electric potential (usually ground) as a reference electrode (shown on top plate in Fig. 2) in a reference state and the electrode supplying the driving electric potential as the driving electrode in a driving state. Although the reference electrode and the driving electrode can be situated on the same plate, (i.e., the other plate has no electrode) we found that vertically opposing the reference electrode and the driving electrode induces more efficient EWOD actuation. Note, a smaller space "D" (Fig. 2(b)) between two drive electrodes is desirable to have smooth droplet movement with lower driving electric potential.

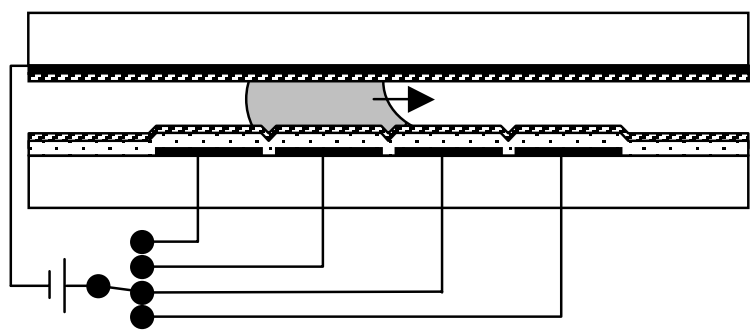

Conductive material (electrodes)

Dielectric layer

Hydrophobic coating

Glass plate

Liquid

(a) Cross sectional view

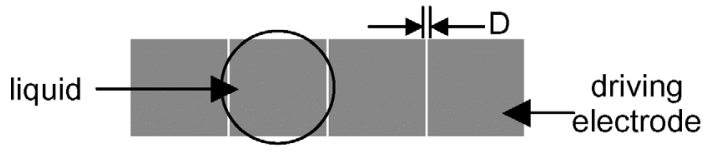

(b) Top view. The blank reference electrode on top plate is not shown.

Figure 2. Single-layer electrode EWOD device.

To reduce fabrication complexity and sustain predictable EWOD actuation, a single conductive layer patterned for electrodes and conduction lines currently presents the most desirable approach. Fig. 3 shows the top view of a possible digital microfluidic circuit applying the electrode configuration previously described in Fig. 2 into the conceptualization depicted in Fig. 1. As the number of electrode increases, the number of conduction lines connecting the outside control circuits to the inner electrodes, likewise increase. These lines must run through 
the small spacing "D" between electrodes with single conduction layer architecture. Since "D" is limited for efficient droplet actuation, the number of drive electrodes is also limited. Moreover, if each drive electrode is controlled by an individual control signal, the complexity of the electric circuit escalates when dealing with a large electrode grid.

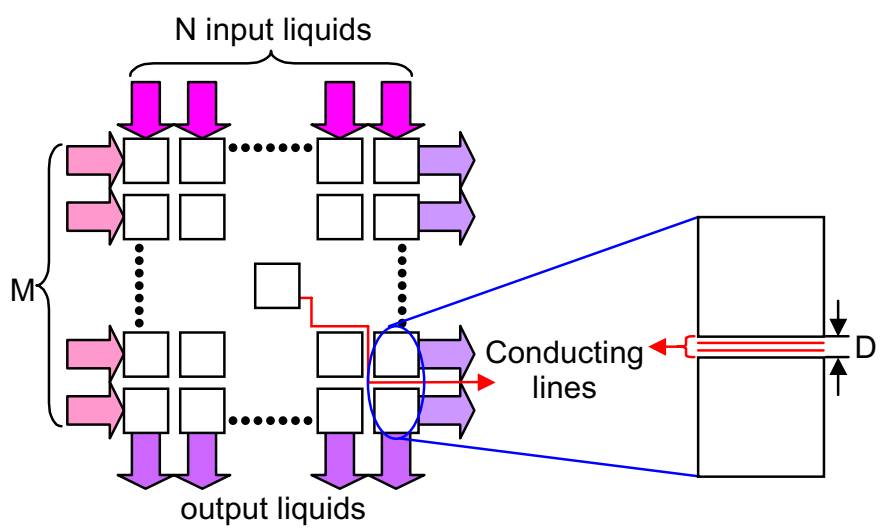

Figure 3. Top view of digital microfluidics circuit with $\mathrm{N}+\mathrm{M}$ input liquids.

\section{THEORY}

Observation of a droplet moving on a 2-D grid, as shown in Fig. 4, revealed the possibility of dividing the movement into two components: (1) X-direction and (2) Y-direction. Therefore, our approach is to position the droplet by using two groups of electrodes. One set of electrodes control the X position of the droplet, while the other set controls the Y position. This concept is similar to a $\mathrm{XY}$ table with two drive motors.

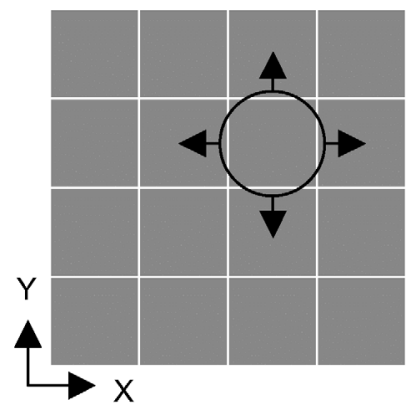

Figure 4. Droplet movement in a $4 \times 4$ electrode array

To achieve this concept, two sets of orthogonal electrode rows are required, one row of electrodes on each top and bottom plate. As shown in Fig. 5, each row is responsible for one direction movement. For example, movement in the X-direction can be made by the rows of electrodes on bottom plate and movement in the Y- direction by the rows of electrodes on top plate. As described previously in Fig. 2, one reference electrode is kept grounded while potential is applied to the individual drive electrodes to drive the droplets. The same driving scheme is conserved in this configuration. Since we can independently control the potential of each electrode, we can switch between reference and driving states for each electrode. Electrode(s) within an electrode row on either top or bottom plates are then assigned reference or driving states depending on the direction of movement. The beauty of this configuration lies in the reduced number of control signals. Only $\mathrm{N}+\mathrm{M}$ control signals are necessary to create a virtual NxM grid.
Looking into X-direction:

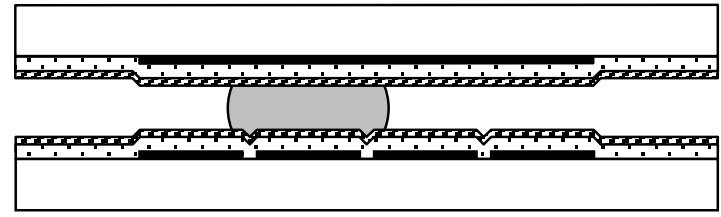

Looking into Y-direction:

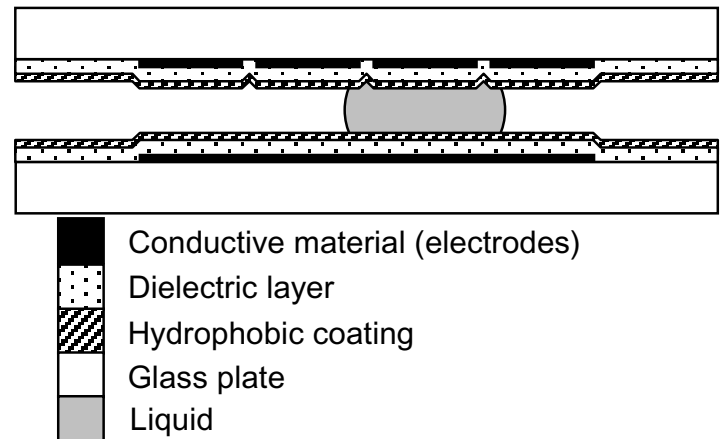

Figure 5. Cross sections of an EWOD device with driving electrodes on both top and bottom channel surfaces.

Note that Figure 2 shows an EWOD device with an asymmetric cross section, while Figure 5 shows one with a symmetric cross section. The contact angle versus applied electric potential measurements of these two situations is discussed in [6]. Experimentally, we verified that EWOD actuation occurs on both asymmetric and symmetric devices.

\section{DESIGN OF ELECTRODE ROWS}

\section{Design I}

Fig. 6 shows the first design. Fig. 6(a) is the top view of a set of electrode rows, which can drive the droplet in the X-direction. These electrode rows consist of vertical major electrodes (four shown) and minor horizontal fingers. Each major electrode branches out to minor electrodes of fingers interdigitating with neighboring electrode fingers. With this design, a droplet sitting on a minor electrode moves only in the X-direction to another minor electrode when activating an adjacent major electrode. This occurs because of the minimized wettable surface above and below each minor electrode. Using the same electrode design in the $\mathrm{Y}$ direction should generate a similar effect (Fig. 6(b)). Initial experiments on the same configuration as in Fig. 2(a) to test EWOD actuation with this electrode row design proved successful. Unidirectional droplet movement was observed when using electrodes within the electrode row (either Fig. 6(a) or 6(b)) as driving electrodes and an unpatterned conductive layer as the reference electrode on the top plate. To test droplet movement in both $\mathrm{X}$ and $\mathrm{Y}$ directions, another set of electrode rows was patterned on separate glass plates and were combined as in Fig. 6(c). However, the anticipated 2-D droplet movement was not obtained. Further investigation revealed that the electric ground through the electrode fingers generated insufficient driving forces. It was found to be important to fill all empty spaces with electrode surface to provide more area for grounding when the corresponding drive electrode is energized. 


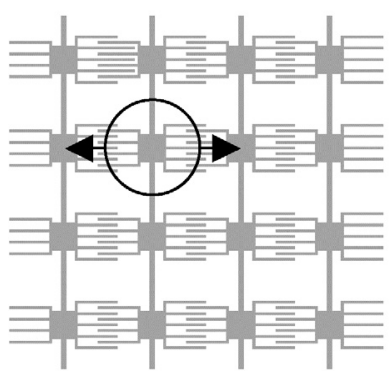

(a) Bottom electrode rows:

$\mathrm{X}$-direction movement.

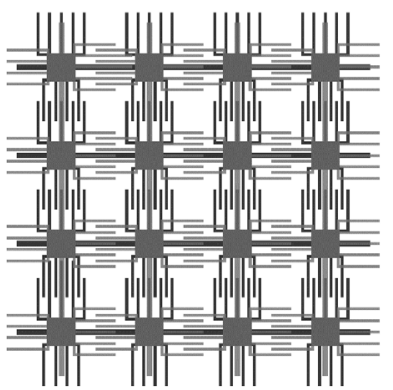

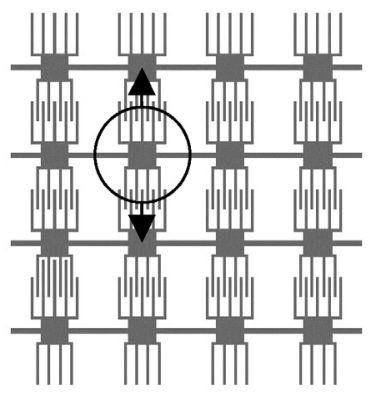

(b) Top electrode rows: Y-direction movement.

(c) left: Orthogonal top and bottom electrode rows: X-Y movement.

Figure 6. Design I: divide driving electrodes into two directions to decrease control signals. X-Y movement is not achieved because of insufficient electric ground.

\section{Design II}

Consequently, an extra surrounding electrode, as shown in Fig. 7, was placed within the area between electrodes in Design II. When the droplet is driven in the X-direction, the actuation scheme is as follows: vertically oriented major electrodes, shown in Fig. 7(a), work as driving electrodes, while all horizontally oriented major electrodes (Fig. 7(b)) on the top plate and the surrounding electrode provide sufficient electric ground. Movement in the Y-direction simply involves reversing this actuation scheme. Later actuation tests successfully demonstrated 2-D droplet movement.

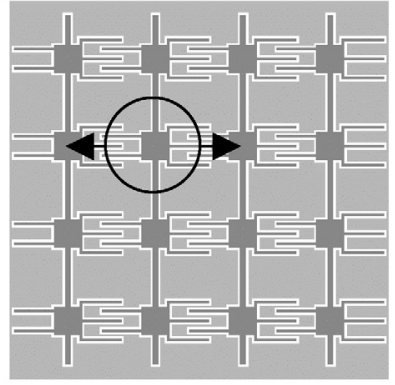

(a) Bottom electrode rows: surrounding electrode is placed to fill between the electrodes. Electrode row is used to drive the droplet along the arrows.

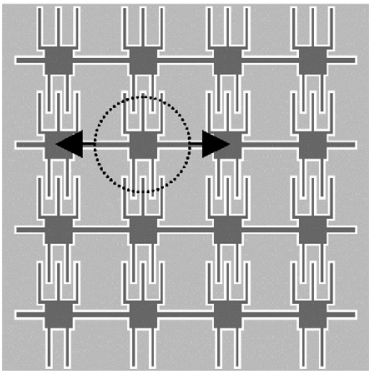

(b) Top electrode rows: both the electrode row and surrounding electrode provide electric ground when droplet moves in the arrow direction
Figure 7. Design II: surrounding electrode is added to provide sufficient electric ground; actuation scheme for droplet movement in X-direction.

By adding a surrounding electrode, we increased the reference electrode area and fulfilled 2-D EWOD actuation. However, the surrounding electrode also decreased the number of fingers between minor electrodes, comparing Fig. 6 and 7. When the number of electrode fingers decreases, the length of the droplet meniscus that contributes to actuation also decreases, resulting in a reduction of EWOD actuation force.

\section{Design III}

The fingers in Design I and II were designed for facilitating droplet movement. The finger design was first introduced by Pollack et al. [7]. However, it was proven (to be reported elsewhere) that, in most cases, droplets can be driven more effectively without the presence of overlapping fingers. Fig. 8 shows redesigned major electrodes with minor electrodes free of fingers. This design maximized overlapping meniscus length thereby theoretically increasing the EWOD actuation force. Testing on this third electrode design indeed resulted in a lower initial driving voltage or higher droplet velocity, compared with Design I and II.

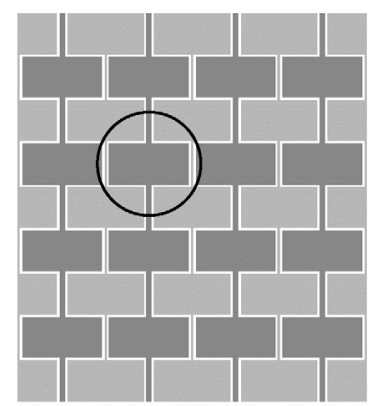

(a) Bottom electrode rows: $\mathrm{X}$-direction movement

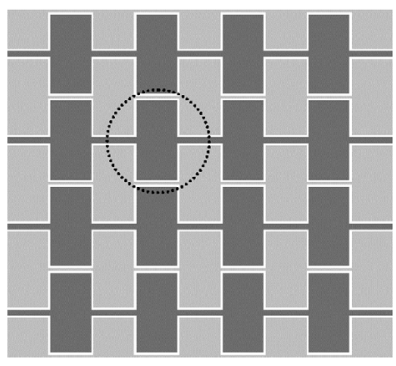

(b) Top electrode rows: Y-direction movement
Figure 8. Design III: electrodes without fingers to provide more electrowetting force. Lower applied voltage or higher moving speed is achieved.

\section{Design IV: Current design (rectangular electrode rows)}

Using the knowledge accumulated through the previous experiments and electrode designs, the current electrode design became elegantly simple. From previous designs, we observed two important factors to design the driving electrode: (1) electrode fingers are not required, (2) maximize length of meniscus overlapping neighboring electrode. The current design, shown in Fig. 9 with rectangular electrodes, is the extreme case with these two factors. It may first appear, with this design, that it will be difficult to confine the droplet movement along a well-define line (Fig 9(a)). However, if only the rectangular electrode right above the droplet is grounded (dash lined in Fig. 9(b)), the movement in the X-direction follows the ground electrode. In short, when one plate (e.g., bottom plate) is used to drive the droplet (e.g., to Xdirection), one of the electrode rows on the other plate (e.g., top plate) can be used to maintain the movement in course (e.g., against Y-direction).

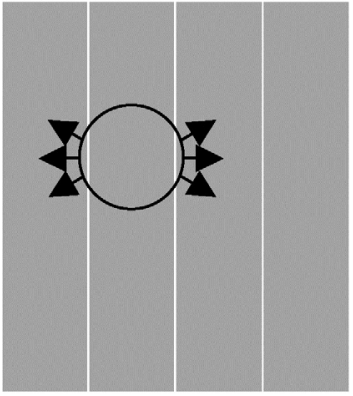

(a) Bottom electrode row: changing the $\mathrm{X}$ position of the droplet. The X-direction movement is not ideal.

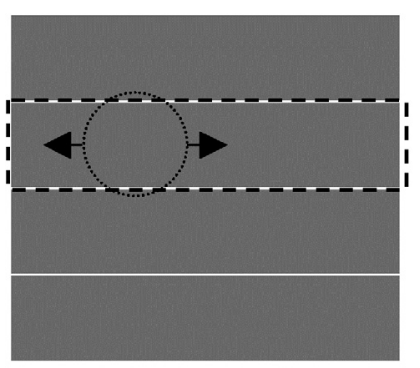

(b) Top electrode row: selectively apply electric ground can keep the Y position of the droplet. 


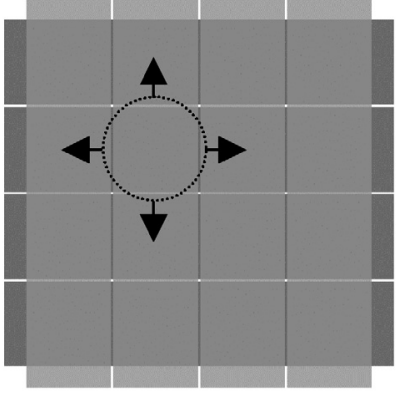

(c) left: 2-D movement of droplet between two electrode sets can be driven by the lowest voltage and the least control signals.

Figure 9. Design IV: Current design using rectangular electrodes.

\section{FABRICATION}

The device for each design consisted of two glass plates with a single layer of patterned electrodes. The electrode top view for each device was shown in the previous figures. Although each design has different electrode shapes, all these designs have the same cross section, as shown in Fig. 5. Since electrodes are placed on both the top and bottom plates, to observe the droplet movement, we required the use of ITO (indium tin oxide), a transparent conductive material deposited and patterned on the top plate. The fabrication processes are as follows: (1) Deposit and pattern ITO (top plate) and $\mathrm{Cr} / \mathrm{Au}$ (bottom plate). (2) Deposit and pattern wire soldering pads on ITO. (3) Deposit dielectric material on both plates. (4) Spin Teflon on both plates. (5) Solder wires to soldering pads.

\section{RESULT}

Fig. 10(a) shows a frame of video recording, which verifies the proposed driving scheme, along with the schematic interpretation of the device. The droplet had a velocity of $30 \mathrm{~mm} / \mathrm{s}$ at $80 \mathrm{~V}_{\mathrm{AC}}$. Higher driving speed is obtainable by applying higher voltage. In this experiment, our goal was to verify the device design; thick $(\sim 0.5 \mathrm{~m})$ dielectric layer is used. By using thinner dielectric layers, driving voltage can be decreased [8].

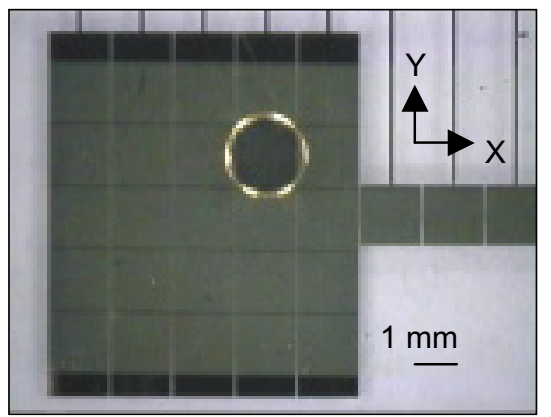

(a) Droplet is moving on a $5 \times 5$ grid.

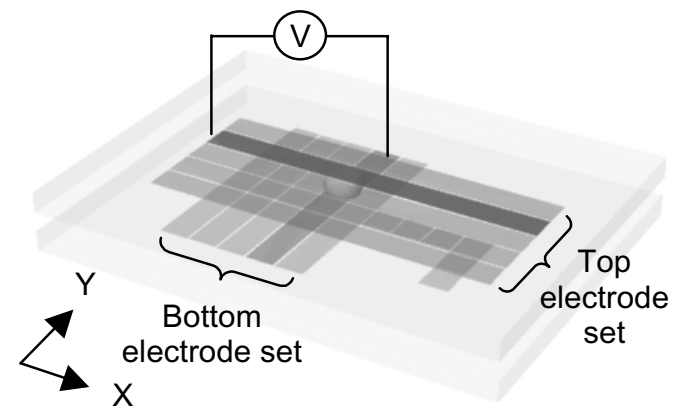

(b) The corresponding activated electrodes of (a) are highlighted.

Figure 10. Testing of the current design.
To demonstrate the programmability, we have driven the droplet to follow alphabets. Fig. 11 shows overlapping images of a droplet traveling along the paths of characters $\mathrm{HH} 02$.
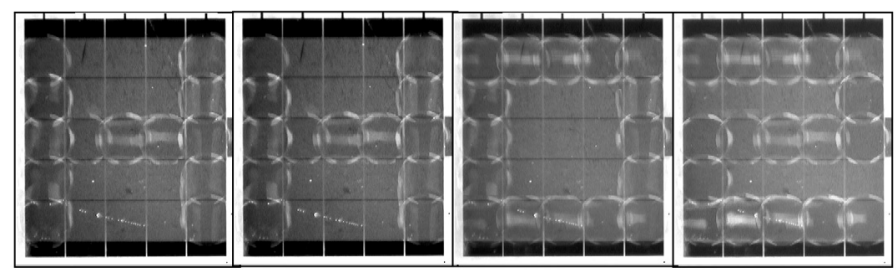

Figure 11. A droplet following characters HH02, demonstrating the programmability for arbitrary continuous path of a droplet on a NxM grid.

\section{CONCLUSION}

EWOD actuation of droplets on a virtual NxM grid using only $\mathrm{N}+\mathrm{M}$ control signals was presented. The current rectangular electrode row design requires only a single conduction layer, thereby simplifying fabrication and minimizing complexity of electrode wiring. The rectangular electrode design improved actuation efficiency by increasing the droplet meniscus length. The result is a major step toward full realization of programmable digital microfluidic circuits.

\section{ACKNOWLEDGEMENT}

This work was supported by the National Science Foundation (NSF) CAREER Award, NSF Engineering Microsystems: "XYZ on a chip" Program, and Defense Advanced Research Projects Agency (DARPA) BioFlips Program. The authors would like to thank the members of Dr. Robin Garrell's group and Sung Kwon Cho, Jesse Fowler, and Hyejin Moon for valuable discussion.

\section{REFERENCES}

1. S. K. Cho, S.-K. Fan, H. Moon, and C.-J. Kim, "Towards Digital Microfluidic Circuits: Creating, Transporting, Cutting and Merging Liquid Droplets by Electrowetting-based Actuation", Proc. IEEE Conf. Micro Electro Mechanical Systems, Las Vegas, NV, Jan. 2002, pp. 32-35.

2. M. Vallet, B. Berge, and L. Vovelle, "Electrowetting of water and aqueous solutions on poly(ethylene terephthalate) insulating films", Polymer, vol.37, (no.12), June 1996. p.2465-70.

3. H. J. J. Verheijen and M. W. J. Prins, "Contact angles and wetting velocity measured electrically," Review of Scientific Instruments, vol.70, (no.9), Sept. 1999. p.3668-73.

4. J. Lee, H. Moon, J. Fowler, C.-J. Kim, and T. Schoellhammer, "Addressable Micro Liquid Handling by Electric Control of Surface Tension," Proc. IEEE Conf. Micro Electro Mechanical Systems, Interlaken, Switzerland, Jan. 2001, pp. 499-502.

5. C.-J. Kim "Micropumping by Electrowetting", Proc. MEMS, ASME IMECE, New York, NY, Nov. 2001.

6. S. K. Cho, H. Monn, J. Fowler, and C.-J. Kim, "Splitting a Liquid Droplet for Electrowetting-Based Microfluidics," Proc. MEMS, ASME IMECE, New York, NY, Nov. 2001.

7. M. G. Pollack and R. B. Fair, "Electrowetting-based Actuation of Liquid droplets for Microfluidic Applications", Applied Physics Letters, Vol. 77, No. 11, pp. 1725-1726.

8. H. Moon, S. K. Cho, R. L. Garrell, and C.-J. Kim, "Low Voltage Operation of Contact Angle Modulation by Electrowetting-On-Dielectric (EWOD) Principle", being submitted. 